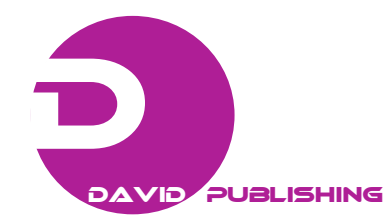

\title{
Cross-Contamination and Transfer Rates of Bacteria on the Cutting Boards
}

\author{
Michiru Kishimoto*, Chiaki Kojima, Ayaka Maeda and Ayako Morinaga \\ 57 Takenoyama, Iwasaki-cho, Nagoya University of Arts and Sciences, Nissin-city, Aichi-pref. 470-0196 Japan \\ *Corresponding author’s e-mail:mkishi@nuas.ac.jp
}

\begin{abstract}
To quantify transfer of bacteria by cross-contamination during cooking operation, the study investigated the rate of transfer from cutting board to ready-to-eat food and other cookware. The study was designed to simulate the real cooking procedures and performed using cutting board that was inoculated with $E$. coli. The cross-contamination contributes to certain fraction of foodborne illness. The recovery rate of swabbing was confirmed by using nonpathogenic Escherichia coli strain JCM1649. Cutting boards, contaminated with different concentrations of $E$. coli, were swabbed by sterilized cotton stick. Transfer rates in the following experiments were calculated by taking into account of the recovery rate, which was 30.5\%. From cutting board, $2.07 \%-3.59 \%$ E. coli was transferred to boiled spinach by cutting procedures. And from cutting board, 0.67\%-1.21\% was transferred to shredded cabbage, $0.11 \%-0.20 \%$ was transferred to kitchen knife or tong by cutting or grasping procedure. This study demonstrated quantitatively the transfer of bacteria from cutting board to cooked spinach, shredded cabbage, knife and tong. The data can be used in quantitative risk assessment of the foodborne pathogens.
\end{abstract}

Key words: Cross-contamination, cutting board, transfer bacteria, quantitative risk. 